\title{
Effects of Grape NAC Transcription Factor DRL1 on Floral Organogenesis in Tobacco
}

\author{
Guirong Li and Ran Quan \\ School of Horticulture and Landscape Architecture, Henan Institute of Science and Technology; and \\ Henan Province Engineering Research Centers of Horticultural Plant Resource Utilization and \\ Germplasm Enhancement, Xinxiang, 453003, China \\ Chaohui Yan \\ College of Horticulture, Northwest A\&F University, Yangling, 712100, China

\begin{abstract}
Xiaojin Hou and Huiling $\mathrm{Hu}$
School of Horticulture and Landscape Architecture, Henan Institute of Science and Technology; and Henan Province Engineering Research Centers of Horticultural Plant Resource Utilization and Germplasm Enhancement, Xinxiang, 453003, China
\end{abstract}

\begin{abstract}
AdDitional INDEX words. flowering time, Nicotiana benthamiana, pollen development, pollen viability, Vitis vinfera
Abstract. Grape (Vitis vinifera) is among the world's most important fruit crops and is a commonly used woody plant for genomics and post-genomics research. NAC transcription factors play central roles in plant growth and development, floral organ morphogenesis, and responses to biological stress. It is therefore important to identify key transcription factors from grape and clarify their mechanisms of action to generate genetic resources for grape molecular improvement. Our research group previously cloned a NAC transcription factor from $V$. vinifera 'Yatomi Rosa' [drought and leaf roll gene 1 (DRL1)] and demonstrated that it caused dwarfing of tobacco (Nicotiana benthamiana) plants when overexpressed. In the present work, we demonstrate that overexpression of $D R L 1$ in transgenic tobacco delays flowering time and markedly reduces pollen viability. Furthermore, crosses between male $D R L 1$ transgenic tobacco and female wild-type tobacco exhibit substantially lower fruit set, fruit and seed weights, fruit and seed shape indices, and seed germination rates than selfed wild-type plants or crosses with a transgenic female parent. DLRI overexpression strongly influences flowering time and reproduction in transgenic tobacco, primarily through its effects on pollen development. These results provide a foundation for further functional characterization of $D L R 1$ in grape.
\end{abstract}

Grape (Vitis vinifera) is a deciduous vine of the Vitaceae family; it is one of the most important fruit crops in the world, with a large cultivation area and a wide variety of uses. It was the first fruit crop with a fully sequenced genome (Jaillon et al., 2007), which led to improvements in fruiting ability and quality; development of new cultivars through genetic engineering; and acceleration of resource evaluation and breeding (Yu et al., 2020).

NACs are a plant-specific transcription factor family whose name is derived from the first letters of the petunia (Petunia hybrida) NAM gene and the Arabidopsis thaliana ATAF1/2 and CUC2 genes (Riechmann et al., 2000). The NAC family is one of the largest gene families in plants (Guo et al., 2015; Hu et al., 2010; Olsen et al., 2005), and its members are involved in various stages of plant growth and development, including the formation of apical meristems (Souer et al., 1996), embryonic

Received for publication 25 June 2020. Accepted for publication 16 Oct. 2020. Published online 13 November 2020.

This work was supported by the Natural Science Foundation of Henan Province China (Grant No. 182300410045).

G.L. and H.H. conceived and designed the experiments. G.L., R.Q., C.Y., and X.H. conducted the experiments and participated in the data analysis, data interpretation, and investigation. G.L. wrote the manuscript. H.H. revised the original manuscript. All authors have read and approved the final manuscript. G.L. is the corresponding author. E-mail: liguirong10@163.com.

This is an open access article distributed under the CC BY-NC-ND license (https://creativecommons.org/licenses/by-nc-nd/4.0/). development (Kunieda et al., 2008), floral organ morphogenesis (Sablowski and Meyerowitz, 1998; Sanders et al., 1999), flowering time regulation (Guo et al., 2018), lateral root initiation (Sohrabi et al., 2017), and leaf senescence (Zhu et al., 2019).

Research on the NAC family in grape has focused mainly on the functional characterization of specific genes (Fernandez et al., 2006; Tello et al., 2015). For example, VpNAC1 from the wild Chinese grape Vitis pseudoreticulata was shown to respond to external pathogens and exogenous hormonal signals, and its overexpression in tobacco (Nicotiana benthamiana) enhanced resistance to powdery mildew (Erysiphe cichoracearum) and Phytophthora parasitica var. nicotianae (Zhu et al., 2012). Seventy-four NAC transcription factors were identified in grape based on a genome-wide analysis, and the functions of a small number of them were demonstrated, mainly regarding stress response and morphological development (Wang et al., 2013). Le Hénanff et al. (2013) found that $V v N A C 1$ was highly expressed in fruit pulp, leaves, and flowers during the late stage of development, suggesting that its function may be related to plant senescence and/or flowering. NAC26 from Vitis amurensis promoted tolerance to drought stress by regulating jasmonic acid in $A$. thaliana (Fang et al., 2016). Various VvNAC26 polymorphisms and their combination influenced berry size variation in grape (Tello et al., 2015). In our previous work, the NAC transcription factor gene DRL1 (GenBank no. XP-002281816) 
was identified from $V$. vinifera 'Yatomi Rosa' and shown to be associated with dwarfing and rolled leaf phenotypes (Yan et al., 2016). DRL1 also appears to have a role in senescence, because its overexpression in tobacco plants significantly delayed leaf senescence (Zhu et al., 2019). Here, we expanded upon this preliminary work by studying the effects of $D R L 1$ on tobacco flowering and fruiting. Specifically, we investigated flowering time, observed the phenotypes of hybrid fruit, and assessed the pollen viability of $D R L 1$ transgenic tobacco. We also transformed tobacco with a DRL1pro::GUS fusion construct to visualize $D R L 1$ overexpression in tobacco floral tissues. The results shed light on the functions of $D R L 1$ and add to our theoretical understanding of NAC transcription factor regulation.

\section{Materials and Methods}

Production of transgenic tobacco. Grapevine is known to be one of the most refractory plants for transformation (Dutt et al., 2007; Duval et al., 2002; Maqsood et al., 2015). Here, we performed heterologous overexpression of $D R L 1$ in transgenic tobacco because of its ease of transformation and regeneration and its short growth period relative to grape. Yatomi Rosa (a very early-maturing red table grape cultivar) was grown in the grape germplasm resource orchard of the Henan Institute of Science and Technology, Xinxiang, Henan, China. The fulllength $D R L 1$ gene was cloned from leaves of 'Yatomi Rosa' and inserted into the fusion GUS gene between Hind III and BamH I of the pCAMBIAP1391Z vector as described in our previous study (Yan et al., 2016). Tobacco transformation was performed as described by Horsch et al. (1985), and the resulting transgenic tobacco plants were selected and self-pollinated to obtain homozygous T2 offspring, which were used for all subsequent analyses. Plants were grown in pots $(15 \mathrm{~cm}$ diameter $\times 13 \mathrm{~cm}$ height) filled with a mixture of nutrient soil, perlite, and vermiculite $(1: 1: 1, \mathrm{v} / \mathrm{v} / \mathrm{v})$ and watered with sterile water in the greenhouse. Greenhouse conditions included a long-day photoperiod (14 h light, $10 \mathrm{~h}$ dark), 25 to $28{ }^{\circ} \mathrm{C}, 2000 \mathrm{~lx}$, and $70 \%$ relative humidity.

Flowering time analysis. Beginning on the first day of flowering, the number of flowers was recorded every $2 \mathrm{~d}$ until the fullbloom stage on 30 plants each of the wild-type and $D R L 1$ transgenic (L1, L3) lines. Data are reported as averages for each genotype.

GuS HISTOCHEMICAL ANALYSIS. For histochemical staining of GUS activity, samples were vacuum infiltrated with GUS staining solution for $30 \mathrm{~min}$ (Roni et al., 2006; Zhu et al., 2019). A tobacco flower develops over $12 \mathrm{~d}$ from a flower bud to a fully bloomed flower. Five different stages of floral development (f1, f2, f3, f4, and f5) of wild-type and DRL1 transgenic tobacco are shown in Fig. 1. Flowers from each stage were stained with GUS, and images of the staining patterns were collected with a microscope and digital camera [10 × 40 (Ds-Fi1C; Nikon, Tokyo, Japan)].

HYBRIDIZATION OF TRANSGENIC PLANTS AND OBSERVATION OF FRUIT CHARACTERISTICS. The teffect of DRL1 on fruit set and other reproductive parameters was investigated in four tobacco genotypes: 1) S1, selfed wild-type tobacco; 2) $\mathrm{C} 1-1$, C1-3, wild-type (ㅇ) $\times D R L 1$ transgenic ( 1$)$ tobacco L1, L3; 3) S2-1, S2-3, selfed DRL1 transgenic tobacco L1, L3; and 4) C2-1, C2-3, DRL1 transgenic tobacco (ㅇ) L1, L3 $\times$ wild-type tobacco ( $\hat{\sigma})$. After pollination, the rate of fruit set was calculated as

fruit set rate $($ percent $)=$ number of fruit

/total number of pollinated flowers $\times 100$

The fruit weight and 1000-seed weight were measured with an analytical balance. A Vernier caliper was used to measure the vertical and transverse diameters of fruit. The fruit shape indice was calculated as

\section{fruit shape index $=$ vertical diameter $/$ transverse diameter}

Thirty seeds were placed in 90-mm plastic petri dishes lined on the bottom with double-folded germination paper. The dishes were arranged on shelves in a growth chamber set at $25^{\circ} \mathrm{C}$ with a 16 -h dark and 8 -h light photoperiod. Seeds were considered to have germinated when a radical had emerged. The seed germination rate was calculated as
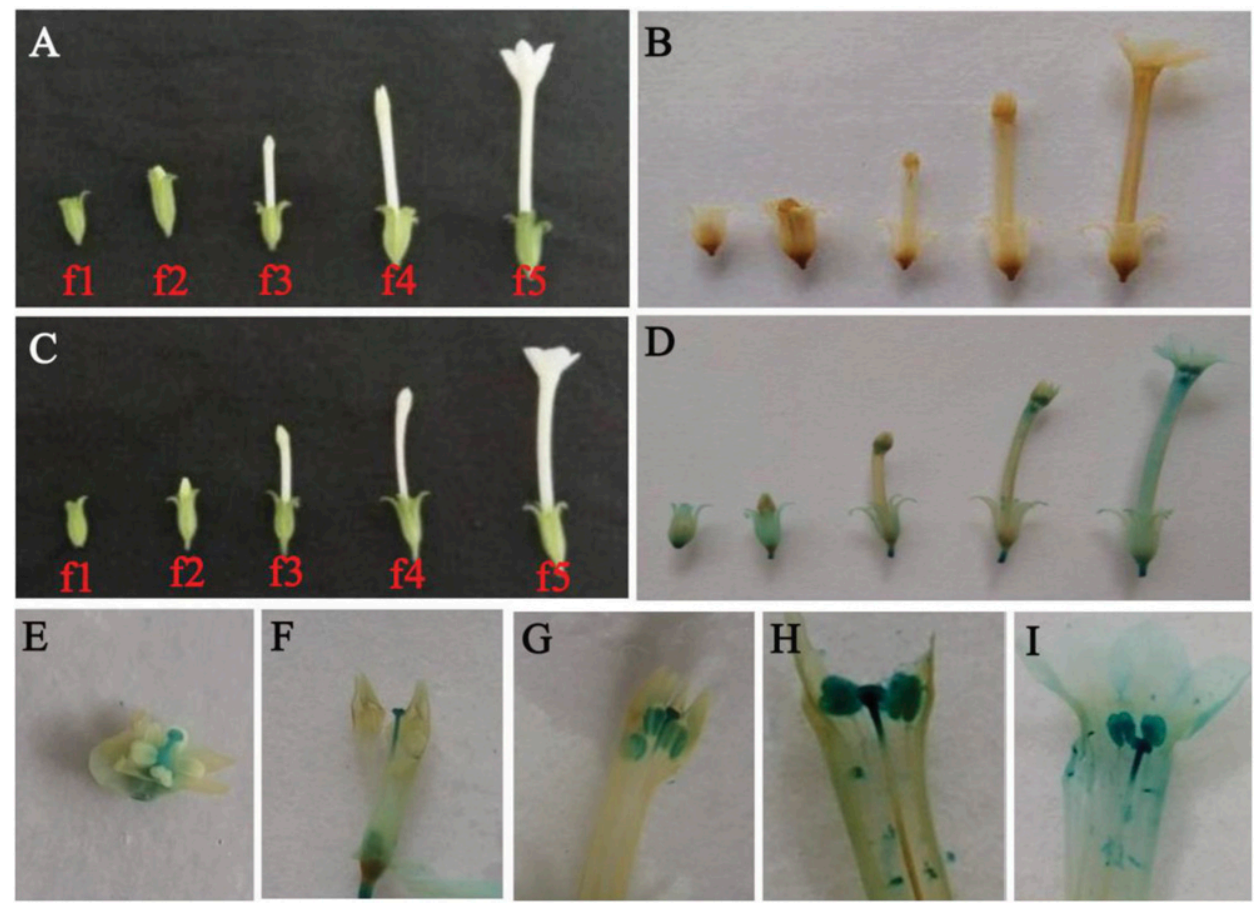

Fig. 1. GUS staining patterns in wild-type and DRL1pro::GUS tobacco plants. (A) Five different stages of floral development in wild-type tobacco. (B) GUS staining of wild-type tobacco at different developmental stages. (C) Five different stages of floral development in transgenic tobacco. (D) GUS staining of transgenic tobacco at different developmental stages. (E-I) Magnified images of GUS staining in transgenic tobacco at different developmental stages. 
seed germination $($ percent $)=$ germinated seeds

/total seeds $\times 100$

Pollen viability determination. Pollen was collected from budding flowers of wild-type and $D R L 1$ transgenic tobacco (L1, L3) in the early flowering stage to measure pollen viability. Pollen grains were hydrated for $24 \mathrm{~h}$ at $28{ }^{\circ} \mathrm{C}$ under sterile conditions, then germinated in plastic petri dishes $(60 \mathrm{~mm}$ diameter) that contained $8 \mathrm{~mL}$ of medium. The nutrient medium was prepared by dissolving $15 \mathrm{~g}$ sucrose, $0.005 \mathrm{~g}$ boric acid, and $0.002 \mathrm{~g} \mathrm{CaCl}_{2}$ in $60 \mathrm{~mL}$ of double-distilled water, then adding 1 $\mathrm{g}$ agar powder and bringing the volume up to $100 \mathrm{~mL}$ at $\mathrm{pH} 5.8$. Pollen grains germinated after $2 \mathrm{~h}$ of culture in a dark chamber at $25^{\circ} \mathrm{C}$. A pollen grain was considered to have germinated when the length of the germinated pollen tube was equal to or longer than the diameter of the pollen. Counts were made at random in three fields under a microscope $[10 \times 40$ (Ds-Fi1C)], and the final germination percentage was defined as

germination $($ percent $)=$ number of germinated pollen grains

/total number of pollen grains $\times 100$

Next, pollen viability was observed using fluorescein diacetate (FDA) staining. FDA mother liquor was prepared by dissolving $2 \mathrm{mg}$ FDA in $1 \mathrm{~mL}$ acetone and stored in an opaque flask at $4{ }^{\circ} \mathrm{C}$. The FDA working solution was prepared by adding $100 \mu \mathrm{L}$ FDA liquid to $9.9 \mathrm{~mL}$ of deionized water. Fresh solutions were prepared for each experiment. Pollen was stained in a working solution for $30 \mathrm{~min}$ before observation. Counts were made at random in three fields, and pollen was photographed under an inverted fluorescence microscope $[10 \times$ 40 (Ti-S, Nikon)]. Pollen viability was calculated as

viable pollen $($ percent $)=$ number of stained pollen grains

/ total number of pollen grains $\times 100$

Statistical Analysis. All values were expressed as the mean \pm SD of three replicates. Data were analyzed by one-way analysis of variance, and differences between wild-type and individual transgenic lines were considered significant at $P<$ 0.05. Analyses were performed with statistical software (SPSS version 19.0 for Windows; IBM Corp., Armonk, NY).

\section{Results}

EFFECT OF DRL1 OVEREXPRESSION ON TOBACCO FLOWERING TIME. Wildtype tobacco began to bloom $36 \mathrm{~d}$ after planting, whereas $D R L 1$ transgenic tobacco lines L1 and L3 began to bloom 58 d after planting (Fig. 2). The number of flowers produced by the L1 and L3 transgenic tobacco lines did not differ significantly from that of wild-type tobacco $(P<0.05)$. These results suggest that $D R L 1$ overexpression delayed tobacco flowering time but did not affect flower number.

Analysis of DRL1 OVEREXPRESSION PATTERnS BY GUS STAINING. Figure 1 shows GUS activity in wild-type and DRL1pro::GUS transgenic tobacco plants. GUS staining was detected in sepals and petals at the first and second stages of floral development (f1 and f2). A reduction in GUS staining was observed in sepals and petals from $\mathrm{f} 3$ to $\mathrm{f5}$, and GUS activity was almost undetectable in later developmental stages. GUS staining was detected in pistils and stamens throughout floral development and increased from fl to f5 (Fig. 1D). Magnified images of the five floral development stages (Fig. 1E-I) revealed strong GUS staining in stigmas and anthers, indicating that DRL1 was highly expressed in these tissues.

EFFECT OF DRL1 OVEREXPRESSION ON TOBACCO FRUIT. Table 1 showed that S1 had the highest fruit set rate of $96.7 \% \pm$ $0.04 \%$. The average fruit set rate of $\mathrm{C} 1$ was only $25.6 \% \pm$ $0.03 \%$ and was significantly lower than that of S1 $(P<0.05)$. Therefore, a transgenic male parent significantly reduced fruit set. The average fruit set rate of $\mathrm{C} 2$ was $91.1 \% \pm 0.03 \%$. The average fruit set rate of S2 was only $27.8 \% \pm 0.03 \%$ and was significantly lower than that of $\mathrm{C} 2(P<0.05)$. Therefore, a transgenic female parent had a minimal effect on fruit set. This result is further confirmed by the fact that the fruit set rate of S1 $(96.7 \% \pm 0.04 \%)$ did not differ significantly from that of C2 $(91.1 \% \pm 0.03 \%)(P<0.05)$.

Differences in fruit weight among crosses followed the same pattern as that observed for fruit set. The fruit weight of S1 was the highest $(0.497 \pm 0.03 \mathrm{~g})$. The average fruit weight of $\mathrm{C} 1$ was only $0.227 \pm 0.02 \mathrm{~g}$ and was significantly lower than that of S1 $(P<0.05)$. Therefore, a transgenic male parent significantly reduced fruit weight. The average fruit weight of $\mathrm{C} 2$ was $0.473 \pm 0.02 \mathrm{~g}$. The average fruit weight of S2 was only $0.256 \pm$ $0.02 \mathrm{~g}$ and was significantly lower than that of $\mathrm{C} 2(P<0.05)$. Therefore, a transgenic female parent had a minimal effect on fruit weight. The same patterns were observed for seed weight, fruit shape indice, and seed germination rate. Furthermore, the cross with a transgenic female parent exhibited normal fruit development and morphology (Fig. 3), but the fruit of the cross with a transgenic male parent were dry and long, with abnormal development. $D R L 1$ overexpression appeared to have a marked effect on anther and/or pollen function but little effect on female reproductive activity.

EFFECT OF DRL1 OVEREXPRESSION ON TOBACCO POLLEN VIGOR. Pollen vigor was measured to further assess pollen 

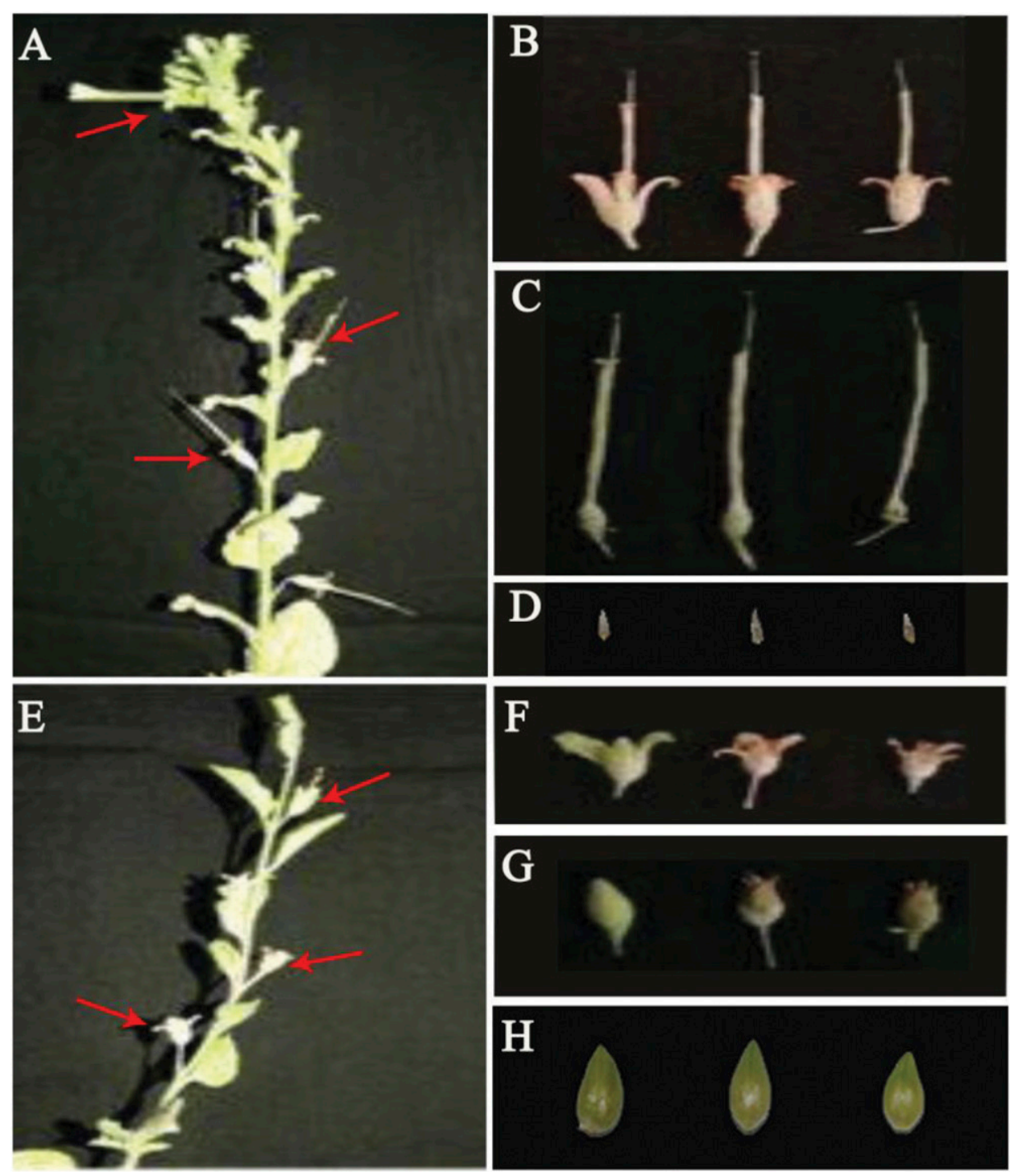

Fig. 3. Fruit set of different genotype crosses of tobacco. (A-D) Phenotypic observations of fruit set in the WT (\$) $\times D R L 1(\widehat{o})$ cross. The fruit were dry and long and exhibited abnormal development. (E-H) Phenotypic observations of fruit set in the $D R L 1(+) \times \mathrm{WT}(\hat{0})$ cross. The fruit exhibited normal development. Red arrows in (A) and (E) indicate developing fruit.

function. Table 2 shows that the in vitro germination percentage of wild-type pollen $(86.84 \% \pm 0.06 \%)$ was significantly greater than that of transgenic pollen $(14.08 \% \pm 0.04 \%)(P<0.05)$. Indeed, little of the transgenic pollen germinated (Fig. 4A and B). We further analyzed the effect of DRL1 overexpression on tobacco pollen vigor using FDA staining (Fig. 4C and D). Over $92 \%$ of the wild-type pollen was viable, whereas only $25.00 \% \pm$ $0.03 \%$ of the transgenic pollen was viable, and this difference was significant $(P<0.05)$.

Taken together, our results suggest that the pollen produced by transgenic plants was much less active and viable than that of wild-type plants, consistent with the differences in fruit set and other reproductive parameters described above.

\section{Discussion}

About 3500 genes are specifically expressed during the development of anthers in higher plants, $60-100$ of which are thought to play key roles in the control of anther development (Sanders et al., 1999). Characterizing the expression of key regulatory genes in anther and pollen development is therefore important to fully understand the molecular mechanisms of pollen development.

The NAC transcription factors (e.g., NTL8, LOV1, OsNTL5, NST, NAP, ATAF1, ATAF2, CUC2, and $A N A C 092)$ constitute a plant-specific transcription factor family with more than 100 members in many terrestrial plant genomes. The NACs are one of the largest transcription factor families in plants and are involved in all stages of plant growth and development, including the development of floral organs. For example, the overexpression of NTL8 in A. thaliana slows plant growth and delays flowering (Kim et al., 2007), whereas the overexpression of LOV1 in A. thaliana causes early flowering (Yoo et al., 2007). OsNTL5 overexpression in rice (Oryza sativa) significantly delays flowering and downregulates the expression of the flowering time regulators Ehdl, Hd3a, and RFTl. Chromatin immunoprecipitation with sequencing (ChIP-seq) results suggest that OSNTL5 binds to the CACG cis-element of the Ehd1 promoter, thereby delaying flowering time through the inhibition of Ehdl expression. Other NACs have been shown to bind directly to the promoter elements of flowering pathway genes to influence flowering (Li et al., 2010). In the present study, the NAC transcription factor $D R L 1$ from $V$. vinifera 'Yatomi Rosa' delayed the flowering time of $D R L 1$ transgenic tobacco plants, consistent with previous studies of NAC genes and flowering time.

NAC transcription factors play important roles in stamen tearing and pollen loosening. For example, the $A$. thaliana NAC transcription factors NST1 and NST2 regulate secondary wall synthesis in pollen sac endothelial cells, thereby controlling stamen rupture (Mitsuda et al., 2005). MYB26 from $A$. thaliana controls stamen secondary wall thickening through its regulation of NST1 and NST2. Likewise, a Tnt1 retrotransposon insertion inhibits alfalfa (Medicago sativa) pollen sac cleavage in an mtnst 1 mutant due to thickening of the secondary wall (Yang et al., 2017). The $A$. thaliana NAC gene $A N A C 092$ is also expressed during specific periods of anther and pollen development ( $\mathrm{Li}$ et al., 2013). In the present study, DRL1pro::GUS transgenic tobacco showed the strongest GUS staining in stigmas and anthers. As floral development 
progressed, stigma and anther staining gradually darkened, indicating that the expression of $D R L 1$ gradually increased during the development of these organs.

Arabidopsis thaliana NARS1/NAC2 and NARS2/NAM belong to the NAC subfamily III. Double mutants exhibit seed shrinkage, but single mutants show no phenotype, indicating that there is functional redundancy between NARS1 and $N A R S 2$. In crosses between wild-type and nars 1 nars 2 double mutants, normal seeds formed when the mutant parent was male, but abnormal seeds formed when the mutant parent was female, suggesting that NARS1 and NARS2 affect ovule development (Kunieda et al., 2008). In the present study, overexpression of $D R L 1$ in tobacco resulted in reduced pollen viability and germination. Likewise, crosses in which DRL1
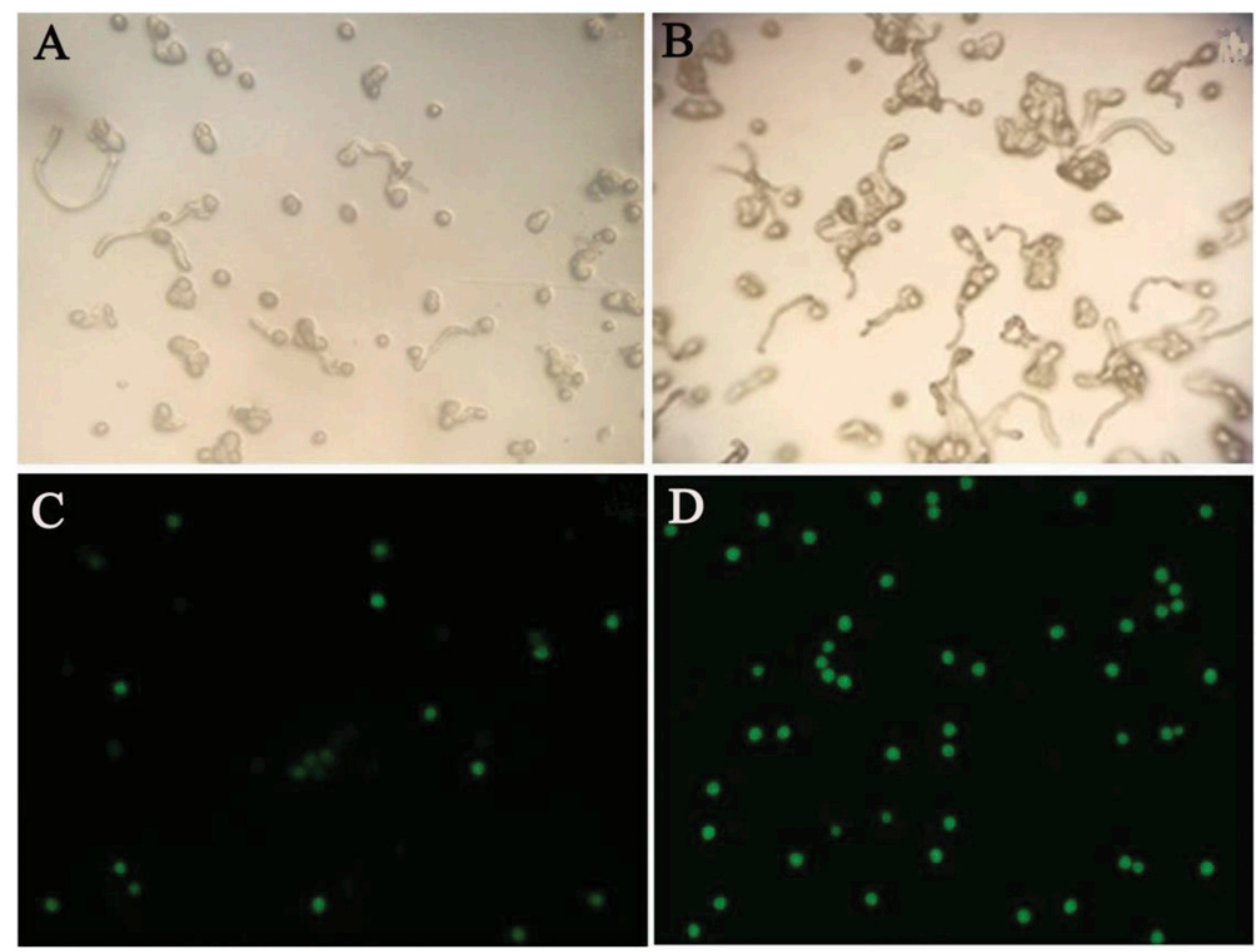

Fig. 4. Pollen viability of $D R L 1$ transgenic tobacco $(\mathbf{A}, \mathbf{C})$ and wild-type tobacco $(\mathbf{B}, \mathbf{D})$ was assessed by an in vitro germination test $(\mathbf{A}-\mathbf{B})$ and FDA staining $(\mathbf{C}-\mathbf{D})$. tobacco was the male parent produced a low fruit set and small fruit, and these effects were not present when the transgenic parent was female. DRL1 appeared to have a significant effect on anther function but a marginal effect on stigma function.

NAC transcription factors are unique to plants, and their functional characterization is a topic of current interest. Their functions are wide-ranging and include the regulation of cell division, flower development, plant senescence, secondary wall growth, lateral root development, stress resistance, and disease resistance (Guo et al., 2018; Hu et al., 2010; Nuruzzaman et al., 2010; Zhong and Ye, 2015). This study focused on changes in flowering time, floral development, and fruit set when the NAC transcription factor DRL1 from 'Yatomi Rosa' grape was overexpressed in tobacco. The specific expression patterns we observed were consistent with a role for DRL1 in the morphogenesis of floral organs in grapevine. Future work will focus on DRL1's effects on lateral root growth, ovule development, disease resistance, and stress resistance, further enriching our theoretical understanding of the regulatory mechanisms of the NAC transcription factors.

\section{Conclusions}

Our results demonstrate that $D L R 1$ overexpression strongly influences flowering time and reproduction in transgenic tobacco, primarily through its effects on pollen development. Given the central role played by NAC transcription factors in plant growth and development, the present work will be of interest to grape researchers for the development of improved germplasm, as well as to researchers with an interest in the function of NAC family transcription factors.

Table 1. Fruit set of different hybridized combinations of wild-type and DRL1 transgenic tobacco.

\begin{tabular}{|c|c|c|c|c|c|}
\hline \multirow{2}{*}{$\begin{array}{l}\text { Hybridized } \\
\text { combination }^{z}\end{array}$} & Fruit set $(\%)$ & Fruit wt $(\mathrm{g})$ & Fruit shape index & Seed wt (g) & Seed germination $(\%)$ \\
\hline & \multicolumn{5}{|c|}{ mean $\pm \mathrm{SD}^{\mathrm{y}}$} \\
\hline$\overline{\mathrm{S} 1}$ & $96.7 \pm 0.04 \mathrm{a}^{\mathrm{x}}$ & $0.497 \pm 0.003 \mathrm{a}$ & $1.55 \pm 0.01 \mathrm{a}$ & $0.089 \pm 0.002 \mathrm{a}$ & $88.3 \pm 0.11 \mathrm{a}$ \\
\hline $\mathrm{C} 1-1$ & $26.5 \pm 0.05 b$ & $0.231 \pm 0.007 \mathrm{~b}$ & $0.91 \pm 0.02 \mathrm{~b}$ & $0.040 \pm 0.003 \mathrm{~b}$ & $30.1 \pm 0.05 b$ \\
\hline $\mathrm{S} 2-1$ & $28.2 \pm 0.02 \mathrm{~b}$ & $0.261 \pm 0.002 \mathrm{~b}$ & $0.81 \pm 0.04 \mathrm{~b}$ & $0.044 \pm 0.003 \mathrm{~b}$ & $33.8 \pm 0.06 \mathrm{~b}$ \\
\hline $\mathrm{S} 2-3$ & $27.3 \pm 0.02 b$ & $0.250 \pm 0.002 \mathrm{~b}$ & $0.77 \pm 0.03 \mathrm{~b}$ & $0.038 \pm 0.003 \mathrm{~b}$ & $32.2 \pm 0.07 b$ \\
\hline $\mathrm{C} 2-1$ & $92.4 \pm 0.04 \mathrm{a}$ & $0.483 \pm 0.003 \mathrm{a}$ & $1.44 \pm 0.05 \mathrm{a}$ & $0.079 \pm 0.006 \mathrm{a}$ & $81.9 \pm 0.14 \mathrm{a}$ \\
\hline
\end{tabular}

${ }^{\mathrm{z}} \mathrm{S} 1$ = selfed wild-type tobacco; C1-1 and C1-3 = wild-type (+) $\times$ DRL1 transgenic tobacco ( $\hat{)}$ ) L1 and L3; S2-1 and S2-3 = selfed DRL1 transgenic tobacco L1 and L3; C2-1 and C2-3 = DRL1 transgenic tobacco ( $($ ) L1 and L3 $\times$ wild-type tobacco ( $)$ ).

${ }^{\mathrm{y}}$ Mean and SD of three independent experiments.

${ }^{\mathrm{x}}$ Within a column, different lowercase letters indicate significant differences between genotypes $(P<0.05$, one-way analysis of variance) 
Table 2. Pollen viability of wild-type and $D R L 1$ transgenic tobacco.

\begin{tabular}{lcc}
\hline & In vitro germination rate & Pollen viability (\%) \\
\cline { 2 - 3 } Genotype & \multicolumn{2}{c}{ mean \pm SD (\%) } \\
\hline Wild-type tobacco & $86.84 \pm 0.06 \mathrm{a}^{\mathrm{y}}$ & $92.45 \pm 0.04 \mathrm{a}$ \\
$D R L 1$ transgenic tobacco L1 & $16.14 \pm 0.05 \mathrm{~b}$ & $26.20 \pm 0.03 \mathrm{~b}$ \\
$D R L 1$ transgenic tobacco L3 & $12.02 \pm 0.03 \mathrm{~b}$ & $23.80 \pm 0.03 \mathrm{~b}$ \\
\hline
\end{tabular}

${ }_{\mathrm{z}}$ Mean and SD of three independent experiments.

${ }^{\mathrm{y}}$ Within a column, different lowercase letters indicate significant differences between genotypes $(P<$ 0.05 , one-way analysis of variance).

\section{Literature Cited}

Dutt, M., Z. Li, S. Dhekney, and D. Gray. 2007. Transgenic plants from shoot apical meristems of Vitis vinifera L. 'Thompson Seedless' via Agrobacterium-mediated transformation. Plant Cell Rep. 26:21012110, doi: 10.1007/s00299-007-0424-6.

Duval, M., T.F. Hsieh, S.Y. Kim, and T.L. Thomas. 2002. Molecular characterization of AtNAM: A member of the Arabidopsis NAC domain superfamily. Plant Mol. Biol. 50:237-248, doi: 10.1023/ a: 1016028530943.

Fang, L., L. Su, X. Sun, X. Li, M. Sun, S.K. Karungo, S. Fang, J. Chu, S. Li, and H. Xin. 2016. Expression of Vitis amurensis NAC26 in Arabidopsis enhances drought tolerance by modulating jasmonic acid synthesis. J. Expt. Bot. 67:2829-2845, doi: 10.1093/jxb/ erw122.

Fernandez, L., A. Ageorges, and L. Torregrosa. 2006. A putative NAP homolog specifically expressed during grapevine flower and berry development. Vitis 45:51-52.

Guo, S., S. Dai, P.K. Singh, H. Wang, Y. Wang, J.L.H. Tan, W. Wee, and T. Ito. 2018. A membrane-bound NAC-like transcription factor OsNTL5 represses the flowering in Oryza sativa. Front. Plant Sci. 9:555, doi: 10.3389/fpls.2018.00555.

Guo, W.L., S.B. Wang, R.G. Chen, B.H. Chen, X.H. Du, Y.X. Yin, Z.H. Gong, and Y.Y. Zhang. 2015. Characterization and expression profile of CaNAC2 pepper gene. Front. Plant Sci. 6:755, doi: 10.3389/fpls.2015.00755.

Horsch, R., J.E. Fry, and N.L. Hoffmann. 1985. A simple and general method for transferring genes into plants. Science 227:1229-1232, doi: 10.1126/science.227.4691.1229.

Hu, R., G. Qi, Y. Kong, D. Kong, Q. Gao, and G. Zhou. 2010. Comprehensive analysis of NAC domain transcription factor gene family in Populus trichocarpa. BMC Plant Biol. 10:145, doi: 10.1186/1471-2229-10-145.

Jaillon, O., J.M. Aury, B. Noel, A. Policriti, C. Clepet, A. Casagrande, N. Choisne, S. Aubourg, N. Vitulo, C. Jubin, A. Vezzi, F. Legeai, P. Hugueney, C. Dasilva, D. Horner, E. Mica, D. Jublot, J. Poulain, C. Bruyère, A. Billault, B. Segurens, M. Gouyvenoux, E. Ugarte, F. Cattonaro, V. Anthouard, V. Vico, C. Del Fabbro, M. Alaux, G.D. Gaspero, V. Dumas, N. Felice, S. Paillard, I. Juman, M. Moroldo, S. Scalabrin, A. Canaguier, I. Le Clainche, G. Malacrida, E. Durand, G. Pesole, V. Laucou, P. Chatelet, D. Merdinoglu, M. Delledonne, M. Pezzotti, A. Lecharny, C. Scarpelli, F. Artiguenave, M.E. Pè, G. Valle, M. Morgante, M. Caboche, A.F. Adam-Blondon, J. Weissenbach, F. Quétier, and P. Wincker. 2007. The grapevine genome sequence suggests ancestral hexaploidization in major angiosperm phyla. Nature 449:463-467, doi: 10.1038/nature06148.

Kim, S.Y., S.G. Kim, Y.S. Kim, P.J. Seo, M. Bae, H.K. Yoon, and C.M. Park. 2007. Exploring membrane-associated NAC transcription factors in Arabidopsis: Implications for membrane biology in genome regulation. Nucleic Acids Res. 35:203-213, doi: 10.1093/ nar/gkl1068.

Kunieda, T., N. Mitsuda, M. Ohme-Takagi, S. Takeda, M. Aida, M. Tasaka, M. Kondo, M. Nishimura, and I. Hara-Nishimura. 2008. NAC family proteins NARS1/NAC2 and NARS2/NAM in the outer integument regulate embryogenesis in Arabidopsis. Plant Cell 20:2631-2642, doi: 10.1105/tpc.108.060160.
Le Hénanff, G., C. Profizi, B. Courteaux, F. Rabenoelina, C. Gérard, C. Clément, F. Baillieul, S. Cordelier, and S. Dhondt-Cordelier. 2013. Grapevine NAC1 transcription factor as a convergent node in developmental processes, abiotic stresses, and necrotrophic/biotrophic pathogen tolerance. J. Expt. Bot. 64:4877-4893, doi: $10.1093 /$ jxb/ert277.

Li, J., X. Cheng, L.Q. Luo, J. Yu, and F. Ming. 2013. Functions of ANAC092 involved in regulation of anther development in Arabidopsis thaliana. Hereditas 35:913-922, doi: 10.3724/sp.j.1005.2013.00913.

Li, J.Q., J.A. Zhang, X.C. Wang, and J. Chen. 2010. A membranetethered transcription factor ANAC089 negatively regulates floral initiation in Arabidopsis thaliana. Sci. China Life Sci. 53:12991306, doi: 10.1007/s11427-010-4085-2.

Maqsood, A., N. Khan, I.A. Hafiz, N.A. Abbasi, M.A. Anjum, and S. Hussain. 2015. Effect of various factors on the efficiency of Agrobacterium-mediated transformation of grape (Vitis vinifera L.). Intl. J. Plant Res. 28:171-178, doi: 10.5958/2229-4473.2015.00024.5.

Mitsuda, N., M. Seki, K. Shinozaki, and M. Ohme-Takagi. 2005. The NAC transcription factors NST1 and NST2 of Arabidopsis regulate secondary wall thickenings and are required for anther dehiscence. Plant Cell 17:2993-3006, doi: 10.1105/tpc.105.036004.

Nuruzzaman, M., R. Manimekalai, A.M. Sharoni, K. Satoh, H. Kondoh, H. Ooka, and S. Kikuchi. 2010. Genome-wide analysis of NAC transcription factor family in rice. Gene 465:30-44, doi: 10.1016/j.gene.2010.06.008.

Olsen, A.N., H.A. Ernst, L.L. Leggio, and K. Skriver. 2005. NAC transcription factors: Structurally distinct, functionally diverse. Trends Plant Sci. 10:79-87, doi: 10.1016/j.tplants.2004.12.010.

Riechmann, J.L., J. Heard, G. Martin, L. Reuber, C. Jiang, J. Keddie, L. Adam, O. Pineda, O.J. Ratcliffe, R.R. Samaha, R. Creelman, M. Pilgrim, P. Broun, J.Z. Zhang, D. Ghandehari, B.K. Sherman, and G. Yu. 2000. Arabidopsis transcription factors: Genome-wide comparative analysis among eukaryotes. Science 290:2105-2110, doi: 10.1126/science.290.5499.2105.

Roni, A., A. Erez, L. Markus, and I.U. Cornelia. 2006. Role of auxin in regulating Arabidopsis flower development. Planta 223:315-328, doi: 10.1007/s00425-005-0088-9.

Sablowski, R.W. and E.M. Meyerowitz. 1998. A homolog of $N O$ APICAL MERISTEM is an immediate target of the floral homeotic genes APETALA3/PISTILLATA. Cell 92:93-103, doi: 10.1016/ s0092-8674(00)80902-2.

Sanders, P.M., A.Q. Bui, K. Weterings, K.N. Mcintire, Y.C. Hsu, P.Y. Lee, M.T. Truong, T.P. Beals, and R.B. Goldberg. 1999. Anther developmental defects in Arabidopsis thaliana male-sterile mutants. Sex. Plant Reprod. 11:297-322, doi: 10.1007/s004970050158.

Sohrabi, S., A. Ebadi, S. Jalali, and S.A. Salami. 2017. Enhanced values of various physiological traits and $V v N A C 1$ gene expression showing better salinity stress tolerance in some grapevine cultivars as well as rootstocks. Scientia Hort. 25:317-326, doi: 10.1016/ j.scienta.2017.06.025.

Souer, E., A. van Houwelingen, D. Kloos, J. Mol, and R. Koes. 1996. The no apical meristem gene of petunia is required for pattern formation in embryos and flowers and is expressed at meristem and primordia boundaries. Cell 85:159-170, doi: 10.1016/s00928674(00)81093-4.

Tello, J., R. Torres-Pérez, J. Grimplet, P. Carbonell-Bejerano, J.M. Martinez-Zapater, and J. Ibanez. 2015. Polymorphisms and minihaplotypes in the $V v N A C 26$ gene associate with berry size variation in grapevine. BMC Plant Biol. 15:253, doi: 10.1186/s12870-0150622-2.

Wang, N., Y. Zheng, H. Xin, L. Fang, and S. Li. 2013. Comprehensive analysis of NAC domain transcription factor gene family in Vitis vinifera. Plant Cell Rep. 32:61-75, doi: 10.1007/s00299-012-1340-y. 
Yan, C.H., G.R. Li, J.Y. Mu, H.T. Lou, and Z.G. Zhu. 2016. Subcellular localization and functional analysis of a NAC gene $V v D R L 1$ from Vitis vinifera 'Yatomi Rosa'. Acta Hort. 43:643-652, doi: 10.16420/j.issn.0513-353x.2015-0707.

Yang, C.Y., J. Song, A.C. Ferguson, D. Klisch, K. Simpson, R. Mo, B. Taylor, N. Mitsuda, and Z. Wilson. 2017. Transcription factor MYB26 is key to spatial specificity in anther secondary thickening formation. Plant Physiol. 175:333-350, doi: 10.1104/pp.17.00719.

Yoo, S.Y., Y. Kim, S.Y. Kim, J.S. Lee, and J.H. Ahn. 2007. Control of flowering time and cold response by a NAC-domain protein in Arabidopsis. PLoS One 2:e642, doi: 10.1371/journal.pone.0000642. Yu, Y.H., L. Bian, K.K. Yu, S.D. Yang, G.H. Zhang, and D.L. Guo. 2020. Grape (Vitis davidii) VdGATA2 functions as a transcription activator and enhances powdery mildew resistance via the active oxygen species pathway. Scientia Hort. 267:109327, doi: 10.1016/ j.plaphy.2019.09.010.

Zhong, R.Q. and Z.H. Ye. 2015. The Arabidopsis NAC transcription factor NST2 functions together with SND1 and NST1 to regulate secondary wall biosynthesis in fibers of inflorescence stems. Plant Signal. Behav. 10:e989746, doi: 10.4161/15592324.2014.989746.

Zhu, Z.G., G.R. Li, C.H. Yan, L. Liu, Q.T. Zhang, Z. Han, and B. Li. 2019. DRL1, encoding a NAC transcription factor, is involved in leaf senescence in grapevine. Intl. J. Mol. Sci. 20:2678, doi: 10.3390/ ijms20112678.

Zhu, Z.G., J.L. Shi, M.Y. He, J.L. Cao, and Y.J. Wang. 2012. Isolation and functional characterization of a transcription factor $\mathrm{VPNAC1}$ from Chinese wild Vitis pseudoreticulata. Biotechnol. Lett. 34:1335-1342, doi: 10.1007/s10529-012-0890-y. 